\title{
Influence of Rare Earth Ions on the Optical Properties of Tellurite Glass
}

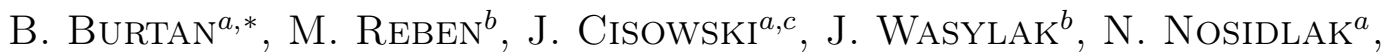 \\ J. JAGLARZ ${ }^{a}$ AND B. JARZĄBEK ${ }^{c}$ \\ ${ }^{a}$ Institute of Physics, Cracow University of Technology, Podchorążych 1, 30-084 Cracow, Poland \\ ${ }^{b}$ Faculty of Materials Sciences and Ceramics, AGH - University of Science and Technology \\ al. A. Mickiewicza 30, 30-059 Cracow, Poland \\ ${ }^{c}$ Centre of Polymer and Carbon Materials, Polish Academy of Sciences \\ M. Curie-Skłodowskiej 34, 41-819 Zabrze, Poland
}

\begin{abstract}
The goal of this work was to investigate the influence of rare-earth ions such as $\mathrm{Nd}^{3+}$ and $\mathrm{Er}^{3+}$ on the optical properties of tellurite glass of the $\mathrm{TeO}_{2}-\mathrm{WO}_{3}-\mathrm{PbO}-\mathrm{La}_{2} \mathrm{O}_{3}$ system. The optical studies of the glasses comprised spectrophotometry (reflectance and transmittance) and spectroscopic ellipsometry. The spectrophotometric measurements yield a number of narrow absorption bands which correspond to characteristic transitions between the ground- and consecutive excited states of rare-earth ions. From ellipsometric studies, in turn, the dispersion of the refraction coefficient has been obtained which appears to be practically the same for the tellurite glass matrix and the matrix doped with $\mathrm{Nd}^{3+}$ and $\mathrm{Er}^{3+}$ ions.
\end{abstract}

PACS: 07.60.Fs, 81.05.Pj, 42.70.Ce, 78.66.Jg

\section{Introduction}

Glass finds increasingly wide application in modern science and technology due to its optical properties. In the last decade, an increasing interest in the heavy metal oxide (HMO) glasses, modified with rare earth elements, is observed and an intensive research is conducted with the aim to shift the absorption edge to the mean infrared for technique of laser glasses and fibre optics in this region.

Among the numerous glass hosts, tellurite glass combines the attributes of wide transmission region, good corrosion resistance, low phonon energy, high refractive index and excellent rare earth ions solubility [1-3]. $\mathrm{TeO}_{2}-$ -based glasses are promising materials for use in non-linear optical devices due to their large third-order non-linear susceptibility $[1,2]$ and for the development of fibre and integrated optic amplifiers as well as lasers covering all the main telecommunication bands because they are potential hosts for infrared (IR) emitting rare earth (RE) elements $[4,5]$.

\section{Experimental \\ 2.1. Glass fabrication}

Tellurite glasses of the $\mathrm{TeO}_{2}-\mathrm{WO}_{3}-\mathrm{PbO}-\mathrm{La}_{2} \mathrm{O}_{3}$ system were obtained by melting $25 \mathrm{~g}$ batches in gold crucibles in an electric furnace at temperature of $850^{\circ} \mathrm{C}$ in

* corresponding author; e-mail: burtan_bozena@wp.pl air atmosphere. The crucibles were covered with a platinum plate to avoid vaporization losses. The melts were poured out onto a steel plate forming a layer thickness of 2 to $5 \mathrm{~mm}$, then annealed in the temperature range of $320-340^{\circ} \mathrm{C}$ for $12 \mathrm{~h}$. The chemicals employed with their high $(99.99 \%)$ purity were as follows: $\mathrm{TeO}_{2}$ (60 mol.\%), $\mathrm{WO}_{3}(27 \mathrm{~mol} . \%), \mathrm{PbO}(10 \mathrm{~mol} . \%)$ and $\mathrm{La}_{2} \mathrm{O}_{3}(3 \mathrm{~mol} . \%)$. In order to introduce the rare-earth ions such as $\mathrm{Nd}^{3+}$ and $\mathrm{Er}^{3+}$ into the tellurite glass matrix, the respective oxides of an amount of $0.1 \mathrm{~g}$ have been added to the batch.

\subsection{Optical measurements}

In order to determine the spectral dependences of reflectance $R(\lambda)$ and transmittance $T(\lambda)$ of the investigated samples, a JASCO V-570 double-beam spectrophotometer operating in the range 190-2500 nm was used.

The ellipsometric data were collected with a M-2000 Woollam ellipsometer in the spectral range 190$1700 \mathrm{~nm}$. The ellipsometric angles $\Psi$ and $\Delta$ fulfill the fundamental equation of ellipsometry, namely $\tan \Psi=$ $\left|r_{p}\right|^{2} /\left|r_{s}\right|^{2} \exp (\mathrm{i} \Delta)$, where $r_{p}$ and $r_{s}$ are complex Fresnel reflection coefficients for $p$ and $s$ polarizations, respectively, and $\Delta$ is a phase shift between both polarized waves. Knowledge of $\Psi$ and $\Delta$ allows one to determine not only the dispersion of the optical constants but also roughness $\sigma$ of a glass [6]. The samples have been measured for three angles of incidence, namely $60^{\circ}, 65^{\circ}$ and $75^{\circ}$. To analyze the data, we have combined all the 
angular spectra and we have fitted all the data simultaneously. The data have been analyzed using CompleteEASE 4.1 software.

\section{Results and discussion}

\subsection{Spectrophotometry}

The specular reflectance and transmittance spectra have been gathered for the tellurite glass matrix (sample $\mathrm{BB}$ ) and for the matrix doped with $\mathrm{Nd}^{3+}$ (sample $\mathrm{BBNd}$ ) and $\mathrm{Er}^{3+}$ (sample BBEr). A comparison between undoped- and rare-earth ion doped glasses is shown in Figs. 1 and 2. It can be seen that introduction of small

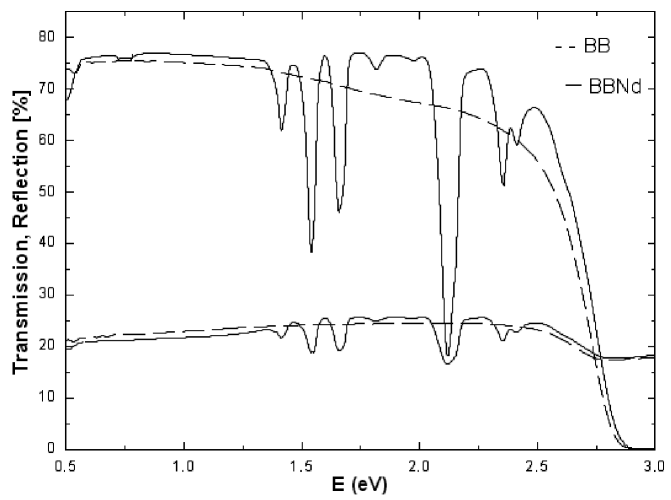

Fig. 1. Specular reflectance and transmittance spectra of the tellurite glass doped with $\mathrm{Nd}^{3+}$ ions (sample BBNd) in comparison to the spectra of the undoped tellurite glass matrix (sample BB).

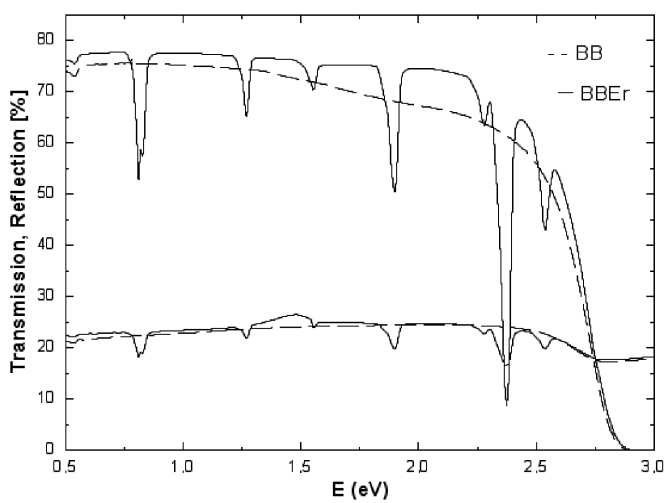

Fig. 2. Specular reflectance and transmittance spectra of the tellurite glass doped with $\mathrm{Er}^{3+}$ ions (sample BBEr) in comparison to the spectra of the undoped tellurite glass matrix (sample BB).

amounts of rare-earth ions results in a dramatic change in the spectra; instead of smooth dependences observed for the undoped sample BB, there is a number of narrow absorption bands, seen not only in the transmittancebut also in the reflectance spectra, which correspond to characteristic transitions between the ground- and consecutive excited states of $\mathrm{Nd}^{3+}[7]$ and $\mathrm{Er}^{3+}$ ions [8].

A comparison of the absorbance spectra of the investigated glass samples is shown in Fig. 3. It can be seen that, above the smooth absorbance of the undoped sample BB, there are sequences of absorption peaks which are quite different for the tellurite glass doped with $\mathrm{Nd}^{3+}$ in comparison to that doped with $\mathrm{Er}^{3+}$ ions. On the other hand, above $2.6 \mathrm{eV}$, all the absorbance curves merge into one which corresponds to the onset of absorption of the tellurite glass matrix.

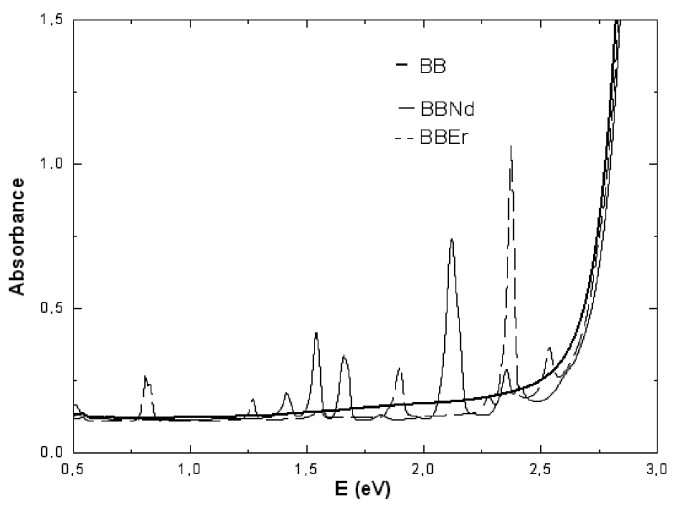

Fig. 3. Absorbance spectra of the tellurite glass doped with $\mathrm{Nd}^{3+}$ (sample BBNd) and $\mathrm{Er}^{3+}$ ions (sample BBEr) in comparison to the spectrum of the undoped tellurite glass matrix (sample BB).

\subsection{Ellipsometry}

The spectroscopic ellipsometry measurements have been performed to get the dispersion relation for the refractive index $n$. The spectroscopic ellipsometry allows also to determine the surface roughness $\sigma$. For our samples we assumed the appearance of the surface roughness which can be described using the Bruggeman effective medium approximation (EMA) [9]. This approximation uses a 50:50 mixture of the material and air at the sample surface to get the optical constants that approximate the effect of the surface roughness. The obtained values of $\sigma$, which are presented in column 5 of Table, appear to be typical for polished glasses.

The spectral dependence $n(\lambda)$ obtained in the range $190-1700 \mathrm{~nm}$ from the fitting procedure for the studied glasses are shown in Fig. 4.

As is seen, the dispersion of the refraction coefficient is practically the same for the samples studied, irrespectively of the absence or presence of rare-earth ions, the fact which strongly influences on the absorption spectra (cf. Fig. 4).

In the region of weak absorption (above $300 \mathrm{~nm}$ ), the Cauchy model of the refractive index dispersion was applied, being expressed by formula

$$
n(\lambda)=A+\frac{B}{\lambda^{2}}+\frac{C}{\lambda^{4}},
$$

where $A, B$ and $C$ are fitting parameters. 


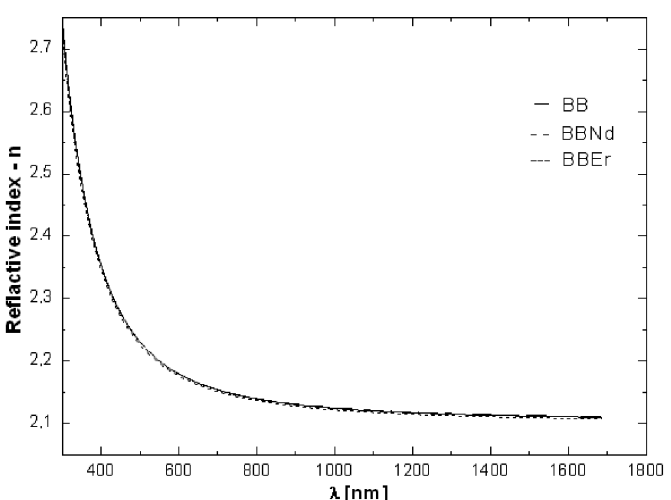

Fig. 4. Dispersion of the refraction coefficient obtained for the investigated glasses from elipsometric measurements.

Values of the Cauchy parameters $A, B$ and $C$ along with the surface roughness determined from the fitting procedure are presented in Table.

TABLE

Cauchy parameters and roughness obtained from ellipsometric studies.

\begin{tabular}{c|c|c|c|c}
\hline \hline Glass & $A$ & $B$ & $C$ & $\begin{array}{c}\text { Roughness } \\
{[\mathrm{nm}]}\end{array}$ \\
\hline BB & 2.092 & 0.01547 & 0.00393 & 10.4 \\
BBNd & 2.104 & 0.01586 & 0.00376 & 6.4 \\
BBEr & 2.102 & 0.01606 & 0.00380 & 8.4
\end{tabular}

The refractive index of tellurite glasses exhibit very high values, over 2.3 , within the $400-450 \mathrm{~nm}$ spectral range which are considerably higher than those obtained for standard optical glasses. The ellipsometric studies have proved that presence of $\mathrm{Nd}^{3+}$ and $\mathrm{Er}^{3+}$ ions do not practically change the refractive index of the tellurite glass matrix.

\section{Conclusion}

In summary, we have shown that the presence of $\mathrm{Nd}^{3+}$ and $\mathrm{Er}^{3+}$ ions in the $\mathrm{TeO}_{2}-\mathrm{WO}_{3}-\mathrm{PbO}-\mathrm{La}_{2} \mathrm{O}_{3}$ glass strongly affects the optical spectra exhibiting a number of narrow absorption bands which correspond to characteristic transitions between the ground- and consecutive excited states of rare-earth ions. On the other hand, as found from the ellipsometric studies, the values and dispersion of the refractive index are practically the same for the undoped tellurite glass matrix and for the matrix doped with $\mathrm{Nd}^{3+}$ and $\mathrm{Er}^{3+}$ ions.

\section{References}

[1] J.S. Wang, E.M. Vogel, E. Snitzer, Opt. Mater. 3, 187 (1994).

[2] T. Kosuge, Y. Benino, V. Dimitrov, R. Sato, T. Komatsu, J. Non-Cryst. Solids 242, 154 (1998).

[3] M. Reben, J. Wasylak, D. Dorosz, Proc. SPIE 7120, 712001 (2008).

[4] H. Chen, Y.H. Zhou, Q.Y. Zhang, Z.H. Jiang, J. Non-Cryst. Solids 351, 3060 (2005).

[5] J. Wasylak, M. Reben, Eur. J. Glass Sci. Techn.: Part B, Phys. Chem. Glasses 48, 264 (2007).

[6] G.E. Jellison, Thin Solid Films 313-314, 33 (1998).

[7] S.S. Babu, R. Rajeswari, K. Jang, C.E. Jin, K.H. Jang, J. Lumin. 130, 1021 (2010).

[8] H.W. Li, S.Q. Man, Opt. Commun. 282, 1579 (2009).

[9] B. Johs, C.M. Herzinger, J.H. Dinan, A. Cornfeld, J.D. Benson, Thin Solid Films 313-314, 137 (1998). 